\title{
Cuentos Americanos
}

\author{
Con Algunos Versos \\ Selected and Edited by Donald D. Walsh \\ Illustrated with pen and ink drawings by Howard Willard
}

Cuentos Americanos presents a lively and altogether readable collection of cuentos and verse that is at once representative of the finest literary production of Latin America and easy enough for classroom use as early as the second semester of first-year Spanish. The list of authors includes many of the most significant and interesting Spanish-American authors of our time.

\section{THE CUENTOS}

Una catta a Dios

El sueño del tejedor

Los tres cuetvos

Historia de dos cachorros de coatí

y de dos cachorros de hombre

De muerte natural

El tatoncito

La voz

El potrillo roano

En las montañas

Contra pereza diligencia

La camisa de Margarita

La lechuza

El buen ejemplo

Los mensú

La Pachacha

Papá y Mamá
Gregorio López y Fuentes (México) Alberto Guillén (Perú)

José Antonio Campos (Ecuador) Horacio Quiroga (Uruguay)

Rafael Bernal (México) Ermilo Abreu Gómez (México)

Arturo Uslar Pietri (Venezuela) Benito Lynch (Argentina)

Ricardo Jaimes Freyre (Bolivia) Ricardo Palma (Perú) Ricardo Palma (Perú)

Alberto Gerchunoff (Argentina) Vicente Riva Palacio (México) Horacio Quiroga (Uruguay) Rafael Maluenda (Chile) Eduardo Bartios (Chile)

In addition there are short poems by Arrieta, Rafael Estrada, Fiallo, Bedregal, Storni, Nalé Roxlo, Balseiro, Gómez Kemp, Alberto Guillén, Lloréns Torres, Ibarbourou, Gabriela Mistral, Dario, Carrera Andrade, Chocano, and Lugones.

210 pages. With notes, exercises, and vocabulary. Price, $\$ 2.25$

"... an introduction to the best in Spanish American letters... has proved to be outstanding in providing worth-while material which can be read by first-year college students with understanding, with interest, and with both literary and linguistic profit."

- Hispania

\section{Examination copies sent upon request}

\section{W - W - NORTON \& COMPANY - INC}




\section{E M O R I A}

DEL

TERCER CONGRESO INTERNACIONAL DE CATEDRATICOS

DE

LITERATURA IBEROAMERICANA

Publicada por

Universidad de Tulane

INSTITUTO INTERNACIONAL DE LITERATURA IBEROAMERICANA

Tomo de más de 250 páginas y 12 trabajos en torno al tema "El nuevo mundo en busca de su expresión"

TRABAJOS :

AUTORES :

La empresa de América y el sentido de la libertad

O homem cósmico de América

Conceitos históricos da América brasileira

Crisis europea, cultura americana

Americanismo y americanidad

México en busca de su expresión

La eternidad de España en América

La democtacia en América

Who speaks for New World Democracy

Posición de América

La expresión literatia de América

La poesía hispanoamericana del presente y del porvenit

José María Chacón y Calvo

Afrânio Peixoto

Gilberto Freyte

César Barja

Baldomero Sanín Cano

Julio Jiménez Rueda

Federico de Onís

Alberto Zum Felde

Henry Seidel Canby

Alfonso Reyes

Antonio Aita

Arturo Torres-Rioseco

Contiene, además, un Prefacio de Arturo Torres-Rioseco

Discursos de los señores

John E. Englekirk

Rufus Carrollton Harris

Carlos García-Prada
Alfred Coester

Mariano Picón-Salas

Noticias sobre otros trabajos y una documentación completa del programa $y$ de las actas del Congreso

$\$ 3.00$ en los Estados Unidos

$\$ 2.00$ en los demás países

Pedidos a:

MIDDLE AMERICAN RESEARCH INSTITUTE

Tulane University

New Orleans, Louisiana 


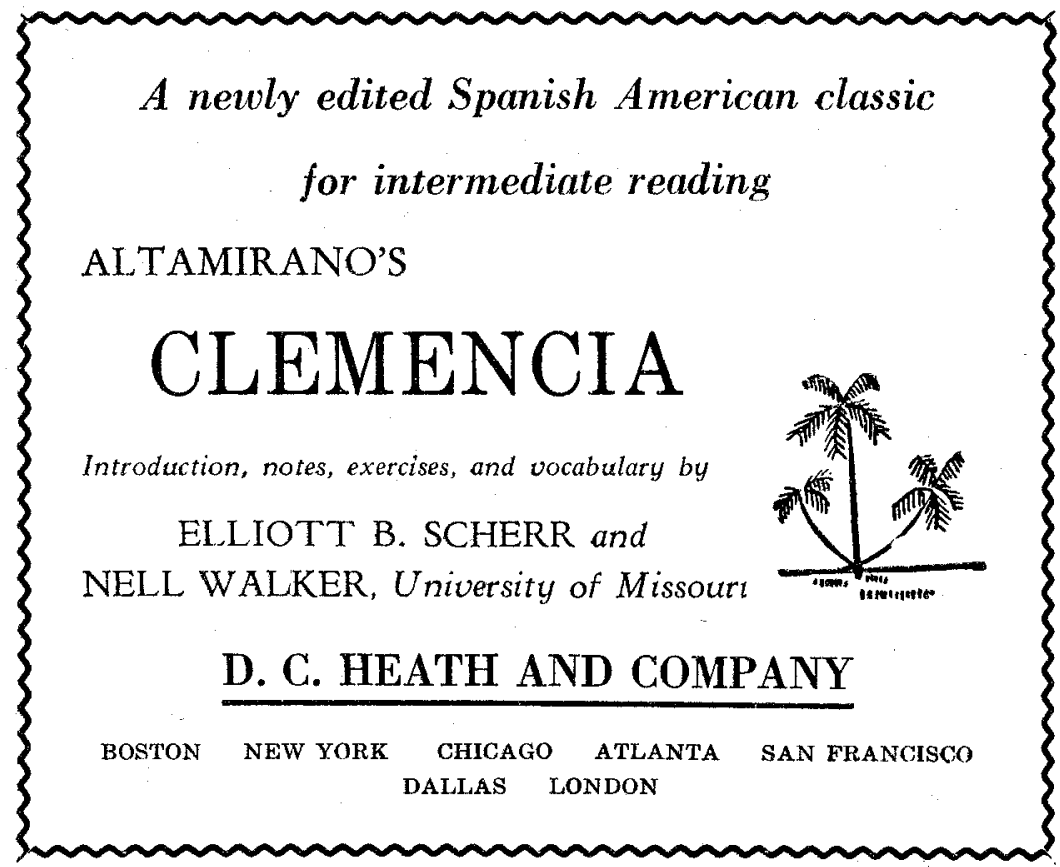

\section{Panorama \\ de las Américas}

\section{$A$ new reader for beginners} by John and Davis Crow

\section{henry holt}

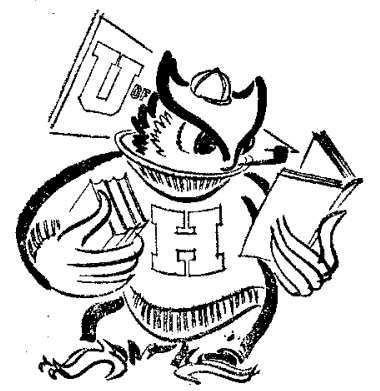




\section{MEMORIA}

OF THE SECOND INTERNATIONAL CONGRESS OF

PROFESSORS OF IBERO-AMERICAN LITERATURE

An excellent collection of studies in Latin American Literature and Philology which contains contributions by many of the most distinguished scholars in the field from Latin America, Spain, and the United States. Only a limited number of copies are available.

A volume of more than 400 pages..........\$3.50

OTHER BOOKS ON HISPANIC SUBJECTS

Grandes novelistas de la América Hispana, with detailed biographical, critical material, and analyses of their works, by Arturo Torres-Rioseco, Professor of Spanish American Literature in the University of California ................ (cloth)

La Novela en la América Hispana, by Arturo Torres-Rioseco ................... (paper)

Don Carlos de Sigüenza y Góngora, a Mexican Savant of the Seventeenth Century, by Irving A. Leonard... $\ldots \ldots \ldots \ldots \ldots \ldots \ldots \ldots \ldots$ (paper)

Spain's Declining Power in Soutb America, the years 1730-1806, by Bernard Moses........... (cloth)

The Civilization of the Americas, by Simpson, Beals, Priestley, Alsberg, González, Fitzgibbon... (paper) Essays in Pan-American, by Joseph B. Lockey. . (cloth) Beside the River Sar: Selections from En las Orillas del Sar by Rosalia de Castro, translated by S. G. Morley ..................... (cloth)

Sonnets and Poems of Anthero De Quental, translated by S. G. Morley................. (cloth)

Studies in the Administration of the Indians of New Spain, by L. B. Simpson............Vol. I \& II

AND OTHERS. WRITE FOR LIST.

ORDERS SHOULD BE SENT TO THE BERKELEY OFFICE

The University of California Press Berkeley and Los Angeles, California 


\section{NUEVO PRECIO DE NUMEROS ATRASAdOS}

DE LA

\section{REVISTA IBEROAMERICANA}

Por el aumento de suscritores que solicitan los primeros números de REVISTA IBEROAMERICANA y la demanda constante de los mismos, por parte de instituciones y particulares que desean tener sus colecciones completas, se hallan a punto de agotarse los números atrasados, que previsoramente se conservaban.

En vista de ello, el Instituto Internacional de Literatura Iberoamericana se ha visto obligado a aumentar el precio de esos números atrasados de la REVISTA, órgano del mismo.

Los precios fijados, por ahora, a los cuatro primeros números, son los siguientes (en dólares):

$\begin{array}{lcc}\text { Número } & \text { Estados Unidos } & \text { Otros países } \\ - & - & - \\ 1 & 2.75 & 2.25 \\ 2 \text { y } 3 & 2.50 & 2.00 \\ 4 & 2.00 & 1.50 \\ 5 \text { y siguientes } & 1.50 & 1.00\end{array}$

Como es fácil advertir por dichos precios, en la venta de esos números atrasados se hacen concesiones análogas a aquellas de que disfrutan los suscritores de la REVISTA IBEROAMERICANA, fuera de los Estados Unidos.

Pedidos a:

JOHN A. CROW

University of California.

Los Angeles, Cal. 


\section{$M E M \bigcirc R I A$}

DEL PRIMER CONGRESO INTERNACIONAL DE CATEDRATICOS DE LITERATURA IBEROAMERICANA

Publicada por

UnIVERSIDAd Nacional INSTITUto INTERNacional DE Autónoma de México Literatura Iberoamericana

Prefacio de Manuel Pedro González UN TOMO DE MÁS DE 200 PÁGINAS, \$1.75

\section{OBRAS COMPLETAS DEL MAESTRO} JUSTO SIERRA

EDICION NACIONAL DE HOMENAJE publicada por la Universidad Nacional de México $y$ dirigida por Agustín YáÑEZ

Volúmenes de que constará la edición:

I. Estudio preliminar y obras poéticas.

II. Teatro y narraciones.

III. Crítica y ensayos literarios.

IV. Periodisno politico.

V. Discursos.

VI. Viajes. En tierra yankee. En la Europa latina.

VII. El Exterior. Revistas políticas y literarias.

VIII. La Educación Nacional. Artículos y documentos.

IX. Semblanzas y ensayos bistóricos.

$\mathrm{X}$. Historia de la antigüedad.

XI. Historia general.

XII. Evolución política del pueblo mexicano.

XIII. Juárez: su obra y su tiempo.

XIV. Epistolario y papeles privados.

$\mathrm{XV}$. Apéndices. Iconografía. Bibliografia. Indices.

Han aparecido los volúmenes IV, V, VI, VII, $\mathrm{x}$, XII $\mathrm{y} \cdot \mathrm{xIII}$. Están por aparecer los restantes. La edición quedará concluída en 1949.

Pedidos a:

UNIVERSIDAD NACIONAL AUTONOMA DE MEXICO

L I B R R I A U I VERSITARIA Justo Sierra, 16

MEXICO, D. F. 


\section{$\begin{array}{llllllllllllll}\boldsymbol{P} & \boldsymbol{U} & \boldsymbol{B} & \boldsymbol{L} & \boldsymbol{I} & \boldsymbol{C} & \boldsymbol{A} & \boldsymbol{C} & \boldsymbol{I} & \boldsymbol{O} & \boldsymbol{N} & \boldsymbol{E} & S\end{array}$ del}

INSTITUTO INTERNACIONAL DE LITERATURA IBEROAMERICANA

\section{Biblioteca de ClÁsicos de AMÉRICA}

Constituirá no sólo una selección de autores y de obras iberoamericanas, sino también una historia de la literatura iberoamericana, en cien tomos. En cada tomo, la selección literaria irá acompañada de un estudio biográfico y crítico, notas explicativas y bibliografía.

Se han publicado los siguientes tomos:

I. Antología poética, de Manuel González-Prada

Estados Otros
Unidos paises

II. Prosas y versos, de José Asunción Silva

III. Cuentos, de Horacio Quiroga

IV. Flor de tradiciones, de Ricardo Palma

V. Don Catrin de la Fachenda, de J. Joaquín Fernández de Lizardi . . 2.50 ” 2.00 ”

\section{Colección literaria, SERIEs A y B}

Amplia y verdadera antología de la poesía iberoamericana contemporánea, editada por Carlos Garcia-Prada. Se publica en dos series. La Serie A es parte integrante de la REVISTA IBEROAMERICANA, órgano del Instituto Internacional de Literatura lberoamericana. La Serie B se publicará en cuadernos separados. Todas las selecciones irán acompañadas de estudios y noticias biográficas y bibliográficas.

De la Serie A se han publicado:

$$
\text { Estados Otros }
$$

Unidos paises

I. 15 poemas, de Porfirio Barba Jacob . $.50 \overline{\mathrm{Dl}} \mathrm{s} . \quad .40 \mathrm{D} l \mathrm{l}$.

II. 16 poemas, de León de Greiff . . .50 " .40 ”

III. 42 pocmas, de Luis C. López . . . . 0 " . .40 ",

IV. 17 poemas, de Julio Vicuña Cifuentes .50" .40 "

V. 35 pocmas, de Rafael Arévalo Marrínez 50 " .40"

VI. 36 pocmas de autores brasileños . . $.50 " .40$ "

VII. 22 poemas, de Arturo Torres-Rioseco .50", .40",

Pedidos a:

JOHN A. CROW

University of California.

Los Angeles, Cal. 
THE SPANISH AND PORTUUGESE TEACHERS' JOURNAL H I S P A N I A

Established 1917

AURELIO M. ESPINOSA, Editor 1917-1926;

ALFRED COESTER, Editor 1927-1941

Published by the American Association of Teachers of Spanish and Portuguese

Editor, Donald Walsh, The Choate School,

Wallingford, Connecticut.

Associate Editors, WILliam BERRIEn, MiChaEl S. DONLAN, AureLIO M. Espinosa, JR., E. HERMAN HESPELT, EDDIE RUTH HUTTON, MARJORIE JOHNSTON, WALTER T. PHILLIPS, STEPHEN L. PITCher, Florence Hall SENDER.

Advertising Manager, DONALD D. WALSH, The Choate School. Wallingford, Connecticut.

HISPANIA appears four times a year, in February, May, October, and December. Subscription (including membership in the Association). $\$ 2.00$ a year; foreign countries, 40 cents additional for postage. Each number contains practical and scholarly articles for teachers of Spanish and Portuguese, including helpful hints for teachers new to the field. $A$ sample copy will be sent on request to the Secretary-Treasurer of the Association. Addres subscriptions and inquiries about membership to: Graydon S. DELAND, Secretary-Treasuret, American Association of Teachers of Spanish and Portuguese, Denison University, Granville, Ohio.

HISPANIA is an ideal medium through which to reach the organized Spanish and Portuguese teachers of the United Satates. For advertising rates, address the Business Manager.

Articles, news notes, and books for review should be addressed to the Editor.

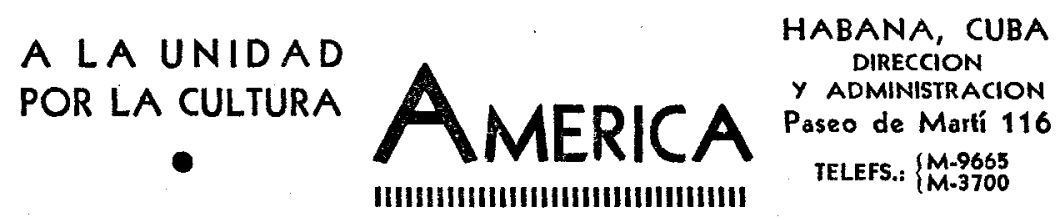

REVISTA DE LA ASOCIACION DE ESCRITORES Y ARTISTAS AMERICANOS

PRECIO DE SUSCRIPCION $\$ 2.00$ DOLARES 


\section{TEXTBOOK NEWS}

\section{An Anthology of Spanish American L I T E R A T UR E}

Hespelt • Leonard • Reid • Crow - Englekirk

A wide selection of the writings of important Spanish American authors, from the colonial period to the present. Biographical and critical introductions. Complete in 1 volume, $824 \mathrm{pp.,}$ $\$ 5.00$; in 2 volumes, each $\$ 3.00$.

\section{An Outline History of Spanish American Literature}

Companion volume by the same authors, $192 \mathrm{pp}$., $\$ 1.75$

APPLETON-CENTURY-CROFTS, INC. 35 West 32nd street

New York 1, New York

\section{LIBRERIA "CERVANTES" DE JULIO SUAREZ}

Iavalle, 558

Buenos Aires

LIBROS ANTIGUOS $Y$ MODERNOS, RA. ROS Y CURIOSOS, REFERENTES A LA AMERICA DEL SUR

Sección especial al servicio de NOVEDADES

(Historia, Literatura, Derecho, Ciencias y Artes)

en las condiciones más ventajosas

Unica agencia de la REVISTA IBEROAMERICANA, en la Argentina

OLD AND RARE LATIN AMERICAN BOOKS
FRANZ C. FEGER

17 E. 22 Street

NEW YORK 10, N. Y.

Américo Castro:

ESPANA EN SU HISTORIA

Cristranos, Moros y Judíos

E1 autor de El pensamiento de Cervantes ha escrito una obra de madurez, fruto de largos años de trabajo. España en su historia es un estudio de incalculable trascendencia, una averiguación completa de "cómo se formó y se desarrolló lo que hoy denominamos la forma hispánica de vida", desde un nuevo punto de vista que antes no había sido tenido en cuenta. De ahí la importancia que otorga a la contextura cristiana-arábigo-judía, desentrañando su influjo en el espíritu y la literatura de la España medieval.

Un volumen, lujosamente encuadernado, de 712 páginas, con láminas ........ U. S. $\$ 10.00$ 
The ANTOLOGIA POETICA of MANUEL GONZALEZ PRADA, first in the series CLASSICS OF LATIN AMERICA to be published under the auspices of the International Institute of Ibero-American Literature, is now for sale at $\$ 2.50$.

The anthology contains nearly 400 pages, is beautifully printed, carries an excellent introduction and many notes by Carlos García-Prada, and is to date the finest single volume representing the works of the famous Peruvian master.

COPIES ARE IIMITED, SO PLEASE PLACE ORDERS AT ONCE WITH JOHN A. CROW, UNIVERSITY OF CALIFORNIA, IOS ANGELES, CAL.

\section{OBRAS POSTUMAS DE GONZALEZ-PRADA}

Trozos de vida (1933) - Poemas ............\$ 100 Bajo el oprobio (1933) - Panfleto contra las tiranías militares en América Latina .............. 0.75 Baladas peruanas (1935) - Poemas ............ 0.50 Anarquia (1936) - Artículos sociales .......... 0.50 Nuevas páginas libres (1937) - Ensayos ......... 0.75 Grafitos (1937) - Epigramas . ............ 1.25 Figuras y figurones (1938) - Artículos políticos .... 0.75 Libertarias $(1938)$ - Poemas ............. 100 Propaganda y ataque (1939) - Artículos religiosos y políticos ...................... 075 Baladas (1939) - Poemas .............. 1.50

\section{De venta en}

LA PRENSA, 245 Canal Street, New York. Para remitir por correo, por cada libro.... 15 centavos " $\quad$ C. O. D. " " " $\quad \ldots 2525$, No envie dinero suelto por correo. - Use cheque o giro postal. 


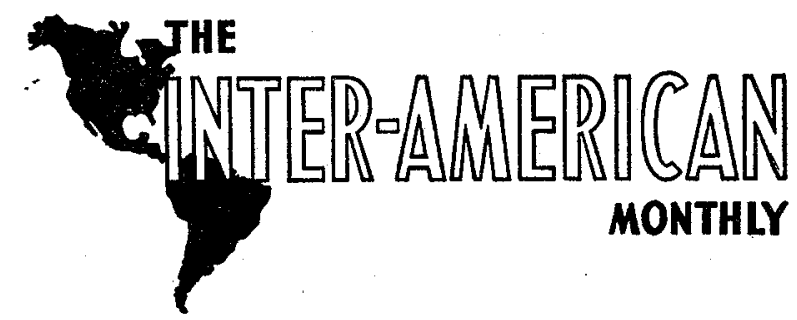

A new publication devoted to reporting and interpreting life in the Americas. Edited by John I. B. MeCulloch, former editor of Pan American News and The Inter-Amer* ican Quarterly - and incorporating both publications.

The Inter-American Monthly is an invaluable source of timely information on politics, headline personalities, art, music, literature, trade and finance, education - comprehensive, authoritative, and realistic.

Free sample copy on request.

Subscription rates: 3 years $-\$ 7,2$ years $-\$ 5$,

1 year $-\$ 3$.

Special rates for classroom use.

\section{THE INTER - AMERICAN MONTHLY}

Washington, D. C.

\section{TULANE UNIVERSITY, colocada estratégi-} camente en $l_{a}$ ciudad de New Orleans, se interesa vitalmente en el desarrollo de una fraternidad más cordial entre las Américas, y por medio de su departamento de español y su Instituto de Middle American Research trabaja hacia este fin. La Universidad saluda al Instituto Internacional de Literatura Iberoamericana como a una organización dedicada al mismo ideal, según se lee en su lema: A LA FRATERNIDAD POR LA CULTURA.

THE TULANE UNIVERSITY OF LOUISIANA

New Orleans 


\section{S Y M P O S I U M}

JOURNAL DEVOTED TO MODERN FOREIGN

LANGUAGES AND LITERATURES

Liferary History.

Comparative Literature.

History of Literary Ideas.

Literature and Society.

Literature and Science.
Philology.

Original Literary Essays.

Trends in Recent Literature.

Notes.

Reviews and Appraisals.

Published twice yearly by the Department of Romance Languages of Syracuse University with the cooperation of the Centro de Estudios Hispánicos and a distinguished board of Associate Editors. $\$ 3.00$ per year.

$\$ 2.00$ per isue.

Albert D. Menut, Chairman Editorial Board.

Albert J. George, Review Editor.

Winthrop H. Rice, Business Manager.

Address: 313 Hall of Languages.

Syracuse University. Syracuse 10, New York.

\section{NOTICE TO MEMBERS}

PLEASE patronize our advertisers and thus

contribute to the financial support of your ins-

titute. Our advertisers have splendid collections of Latin American books at prices no higher than you would pay elsewhere. When ordering from them, please mention the REVISTA. THANK YOU 